\title{
Authigenic Iron Minerals Associated with Iron and Methane Coupling in Marine Sediments
}

\author{
V. BOYKO ${ }^{1 *}$, B. HERUT ${ }^{2}$, O. YORSHANSKY ${ }^{1}$, O. SIVAN ${ }^{1}$
}

${ }^{1}$ Ben-Gurion University of the Negev, P.O.B. 653, Beer

Sheva 8410501 , Israel $(*$ correspondence:

valeri.boyko@gmail.com)

${ }^{2}$ Israel Oceanographic and Limnological Research, TelShikmona, P.O.B. 8030, Haifa 31080, Israel

Iron reduction is an important process in carbon mineralizarion. In this study we show, that in marine sediments, significant iron reduction was observed at depths deeper than its classic zone, at the depth of methanogenesis. Iron reduction in methanogenic sediments can induce the precipitation of secondary authigenic minerals, which may change its chemical character and microbial activity. Our study is aimed to provide insights on iron-methane couplings in the sediments of the Southeastern Mediterranean shelf.

Sediment core, approximately $6 \mathrm{~m}$ long, was sampled by a piston corer at $80 \mathrm{~m}$ water depth offshore Haifa (Israel). Geochemical analyses of the porewater (dissolved iron and manganese, sulfur species, dissolved inorganic carbon, methane) were conducted in order to elucidate redox processes. Total and highly reactive iron contents were measured in the solid phase.

The sulfate-methane transition zone (SMTZ) was detected at ca. $2 \mathrm{~m}$ bsf. Iron reduction zone was found in the upper 0.6 $\mathrm{m}$ bsf and in the deep methanogenic zone at $2.6 \mathrm{~m}$ bsf. Based on these profiles, the sediments from the SMTZ and three layers on the methanogenic zone were used for incubation experiments in order to explore the mechanisms of iron reduction and their possible links to methane cycle. The results show evidences for reduction of ferric iron (hydr)oxides with different reactivity in methanogenic sedimentary zone, followed by formation of authigenic iron minerals. 